\title{
Mycobacterium bovis BCG
}

National Cancer Institute

\section{Source}

National Cancer Institute. Mycobacterium bovis BCG. NCI Thesaurus. Code C85546.

An attenuated form of Mycobacterium bovis that is used to generate the Bacillus

Calmette-Guerin vaccine. 